\title{
Investigation of rheological properties of asphalt emulsions
}

\author{
Enhao Zhang, Xiaofei Qi, Liyan Shan* and Dongsheng Li
}

\begin{abstract}
Rheology is critical issue in the workability, stabilization and engineering performance of asphalt emulsions. The objective of this study is to investigate the rheological properties of asphalt emulsions. To achieve this goal, a preshear protocol was introduced and four kinds of asphalt emulsions were tested base on preshear. Firstly, the necessarity of preshear was analyzed and the suitable range of preshear rate and preshear time of the studied asphalt emulsions were given. Then, the rheological properties of asphalt emulsions were studied after preshear. Finally, the palierne model was modified and the storage/loss modulus of asphalt emulsions was accurately predicted by the modified palierne model. The results showed that it's necessary to preshear asphalt emulsions before testing rheological properties. The viscosity, zero shear viscosity, storage and loss modulus of asphalt emulsions increase with the increase in solid content. The prediction results of the modified palierne model are better than that of palierne model. The storage/loss modulus of asphalt emulsions can be effectively predicted by the modified palierne model. This study has considerable meaning to the promotion of production and manufacturing of asphalt emulsions.
\end{abstract}

Keywords: Asphalt emulsions, Rheological properties, Test method, Preshear

\section{Introduction}

As a kind of typical colloidal material, asphalt emulsions have been widely used in the construction, maintenance and repairment of pavement [1]. Rheological properties of asphalt emulsions are usually thought to be the sensitive technical indexes in accurately describing the performance of asphalt emulsions in civil engineering. The production, design, and engineering performance of asphalt emulsions are related to its rheological properties $[2,3]$. Thus, the investigation of its rheological properties is necessary.

Up to now, most studies focus on the rheological properties of residue of asphalt emulsions. The study on rheological properties of asphalt emulsions is limited. Viscosity is the most widely used index to describe engineering performance of asphalt emulsions and it is affected by many factors such as temperature, $\mathrm{pH}$ value,

\footnotetext{
*Correspondence: myshanliyan@126.com

School of Transportation Science and Engineering, Harbin Institute of Technology, Harbin 150090, Heilongjiang, China
}

droplet size, etc.. The viscosity of asphalt emulsions increases with the decrease in temperatures [4]. With the addition of acid or alkali solution, the viscosity of asphalt emulsions also increases $[5,6]$. The viscosity of asphalt emulsions mainly depends on the interactions of droplets. Mercado et al. found that the viscosity of asphalt emulsions increases with the decrease in the average droplets size and decreases when the droplet size distribution is narrow [4]. They also indicated that the viscosity of asphalt emulsions decreases with the addition of sandstone powder [7]. Modifiers can enhance the interactions of droplets of asphalt emulsions and the rheological properties were improved. Dynamic modulus can also characterize the engineering performance of aphalt emulsions. Liu et al. and Yu et al. demonstrated that the dynamic modulus of asphalt emulisons was enhanced by the addition of waterborne epoxy resin and nanoscale polyurethane $[8,9]$. Sarkar et al. found that the rutting indexes of tire-rubber modified asphalt emulsions is higher than that of unmodified asphalt emulsions [10]. Shirkavand et al. found that the G' and G" of 
asphalt emulsions increase with the addition of nanoclay [11]. However, the colloidal system of asphalt emulsions is thermodynamically unstable and the rheology test results could be affected by their mechanical history. Above studies directly test the rheological properties of asphalt emulsions and the effect of mechanical history was ignored. Ouyang et al. presheared the asphalt emulsions and cement asphalt emulsion paste before rheology tests [12-14]. But they presheared different materials with same preshear protocol. The method to determine the preshear protocol was not studied.

In the field of emulsion science, researchers found that rheology test results can be affected by mechanical history of emulsions. Thus, preshear was employed to eliminate this effect [15]. A preshear protocol refers to preshear emulsions for a period of preshear time under a certain preshear rate. Researchers presheared samples with a period of time, and then the rheological properties of the studied emulsions such as clay suspensions, laponite suspensions, etc. were tested [16-20]. Pignon et al. considered preshear as a proper procedure for thixotropy testing of suspensions [16]. Sun et al. demonstrated that rheology test results of Laponite suspensions have good repeatability after preshear [17]. Rogers et al. and Thomas et al. [18-20] also proved the necessity of preshear during the rheology test of colloidal glasses and colloidal gel. Some researchers found that residual stress will exist in emulsions after preshear and the emulsions cannot be immediately tested after preshear. Thus, the recovery time was used to eliminate the effect of residual stress. Conte et al. found that the rheology test results of cement pastes with $60 \mathrm{~s}$ recovery time is reliable [21]. Vincent et al. showed that the attractive gels need a recovery time of $20 \mathrm{~s}$ after preshear [22]. Asphalt emulsion is also a kind of emulsion. The study on preshear is helpful to the accurate characterization of rheological properties of asphalt emulsions.

The rheological properties of asphalt emulsions are determined by the interaction force among dispersed droplets which was affected by droplet size and droplet size distribution $[15,23]$. The establishment of the relationship between rheological properties and microstructures will be a great promotion to the design and production of asphalt emulsions. Studies proved that the viscosity of asphalt emulsions was affected by droplet size and droplet distribution [7]. The viscosity of asphalt emulsions increases with the increase in average droplet sizes $[15,24-26]$, and decreases with the decrease in droplet size distribution [24-26]. Furthermore, the viscosity of asphalt emulsions with wide droplet distribution curve is larger than others [15, 23, 27]. Except that, Ronald et al. found that the storage and loss modulus decrease with the increase in droplet size
[28]. In the above studies, qualitative analysis or simply regression was used to study the relationship between microscopic characteristics and rheological properties of asphalt emulsions. A model which can predicted the dynamic modulus of asphalt emulsions based on microscopic characteristics is lacked. Palierne proposed Palierne model which can predicted the dynamic modulus of emulsions based on microscopic characteristics [29]. Through Palierne model, Themeli et al. studied the effect of asphaltite on dynamic modulus of asphalt binders [30]; Tan and Guo studied the effect of filler volume on interaction parameter of asphalt mastics [31]. Up to now, Palierne model has not been applied on the study of asphalt emulsions.

The objective of this study is to investigate the rheological properties of asphalt emulsions. Firstly, the necessity of preshear was analyzed and the preshear protocol was investigated. Then, the rheological properties of asphalt emulsions were tested after preshear. Finally, the storage/loss modulus of asphalt emulsions was successfully predicted by the modified palierne model. The research in this study reveals the viscoelastic behavior of asphalt emulsions, and is helpful to the design and production of asphalt emulsions.

\section{Experimental procedures}

\section{Materials and sample preparation}

At present, there are four kinds of asphalt emulsions, cationic asphalt emulsions, anionic asphalt emulsions, non-ionic asphalt emulsions and amphoteric asphalt emulsions. The adhesion between cationic asphalt and aggregate is better than that of anionic asphalt emulsions. The cost of cationic asphalt is lower than that of non-ionic asphalt emulsions and amphoteric asphalt emulsions. Cationic asphalt emulsions are widely used in engineering. Slow-cracking asphalt emulsions are more suitable for asphalt mixtures. Thus, rheological properties of cationic asphalt emulsions were studied in our study. A $60 \sim 80$ grade asphalt binder A is used as disperse phase for emulsions fabrication, whose general characterization is reported in Table 1. Cetyltrimethylammonium bromide is used as emulsifiers to fabricate emulsions A-40, A-50 and A-60, whose solid contents are 40,50 and $60 \%$ respectively. Asphalt emulsion B is a kind of commercial asphalt emulsion whose solid content is $40 \%$. The basic performances of the studied asphalt emulsions are shown in Table 2.

Table 1 Basic performances of neat asphalt binder

\begin{tabular}{llll}
\hline Type & $\begin{array}{l}\text { Penetration } \\
\left(\mathbf{1 0 0 ~} \mathbf{g}, \mathbf{2 5} \mathbf{5}^{\circ} \mathrm{C}, \mathbf{5 ~ s},\right. \\
\mathbf{0 . 1} \mathbf{~ m m})\end{array}$ & Soft point $\left({ }^{\circ} \mathrm{C}\right)$ & Ductility $\left(\mathbf{1 5 ^ { \circ } \mathrm { C } )}\right.$ \\
\hline Asphalt binder A & 68.0 & 49.0 & $>100$ \\
\hline
\end{tabular}


Table 2 Basic performances of the studied emulsified asphalt binders

\begin{tabular}{|c|c|c|c|c|}
\hline \multirow[t]{2}{*}{ Property } & \multicolumn{4}{|c|}{ Measured values } \\
\hline & $A-40$ & $A-50$ & A-60 & B \\
\hline Ion type & Cationic & Cationic & Cationic & Cationic \\
\hline $\begin{array}{l}\text { Emulsified breaking } \\
\text { rate }\end{array}$ & Slow crack & Slow crack & Slow crack & Slow crack \\
\hline $\begin{array}{l}\text { Remaining amount on } \\
1.18 \mathrm{~mm} \text { sieve }(\%)\end{array}$ & 0.075 & 0.025 & 0.05 & 0.08 \\
\hline Viscosity (Pa•s) & 4 & 5 & 7 & 6 \\
\hline Residue content (\%) & 40.07 & 50.40 & 60.06 & 40.10 \\
\hline \multicolumn{5}{|l|}{ Storage stability (\%) } \\
\hline $1 d$ & 0.32 & 0.44 & 0.29 & 0.06 \\
\hline $5 d$ & 3.35 & 3.21 & 7.89 & 0.08 \\
\hline
\end{tabular}

Before testing, about $2 \mathrm{ml}$ asphalt emulsion was dropped onto the under plate. The gap between upper plate and under plate was adjusted to $55 \mu \mathrm{m}$ for pre-compaction. In order to prevent sample splashes and water evaporation during the test, a material is needed to seal the sample. This kind of material can neither react with asphalt emulsions, nor affect the rotation of cone-plate. Thus, methyl silicone oil with low viscosity was chosen to seal the sample, as shown in Fig. 1b. Finally, the gap between upper plate and under plate was set to $50 \mu \mathrm{m}$ for testing.

\section{Characteristic of rheological performance}

Rheological data were obtained using a DHR-2 rheometer of TA company in this study. A cone-plate configuration of radius $r=25 \mathrm{~mm}$ and angle $\alpha=2^{\circ}$ was chosen, as shown in Fig. 1. Before testing, stess sweep tests were conducted on samples to ensure the linear region. The linear region is defined as the region where the dynamic shear modulus decreases to $90 \%$ of the initial value. Curves of stress sweep were shown in Fig. 2 and the corresponding linear rheological regions were shown in Table 3.

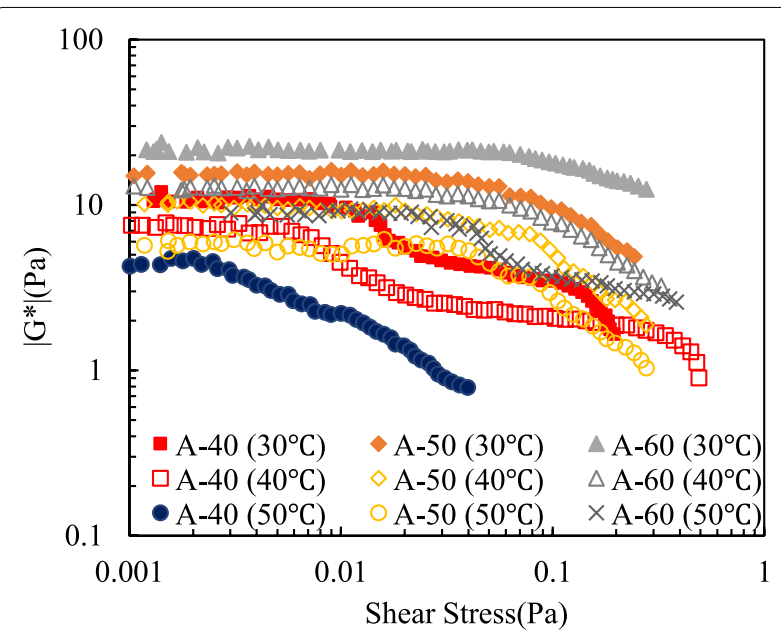

Fig. 2 Results of stress sweep of the studied asphalt emulsions

Viscosity of the studied asphalt emulsions was tested by viscous flow tests at $30{ }^{\circ} \mathrm{C}$. A time sweep test was performed under $0.01 \mathrm{~Pa}$ at $30{ }^{\circ} \mathrm{C}$. Frequency sweep tests were performed at different temperatures ranging from $30{ }^{\circ} \mathrm{C}$ to $50{ }^{\circ} \mathrm{C}$ with an interval of $10{ }^{\circ} \mathrm{C}$. For each test temperature, the test frequencies were set to $0.05 \mathrm{rad} / \mathrm{s}$, $0.1 \mathrm{rad} / \mathrm{s}, 0.2 \mathrm{rad} / \mathrm{s}, 0.5 \mathrm{rad} / \mathrm{s}, 1 \mathrm{rad} / \mathrm{s}, 2 \mathrm{rad} / \mathrm{s}$ and $5 \mathrm{rad} / \mathrm{s}$.

\section{Microscopic properties}

The droplet size distribution of the studied asphalt emulsions was obtained by a laser light scattering equipment (JL-1156). The signal source is an imported semiconductor laser with wavelength of $635 \mathrm{~nm}$. First, distilled water filled $2 / 3$ of the measuring cell and $0.1 \mathrm{ml}$ of dispersing agent was poured into it. Then, 150 times diluted asphalt emulsions were poured into measuring cell. Finally, the mixture of asphalt emulsions, water and dispersing agent is homogenized by ultrasonic dispersion before testing. Each sample was tested for three times at $30{ }^{\circ} \mathrm{C}$ and the results were the average of three replicates.
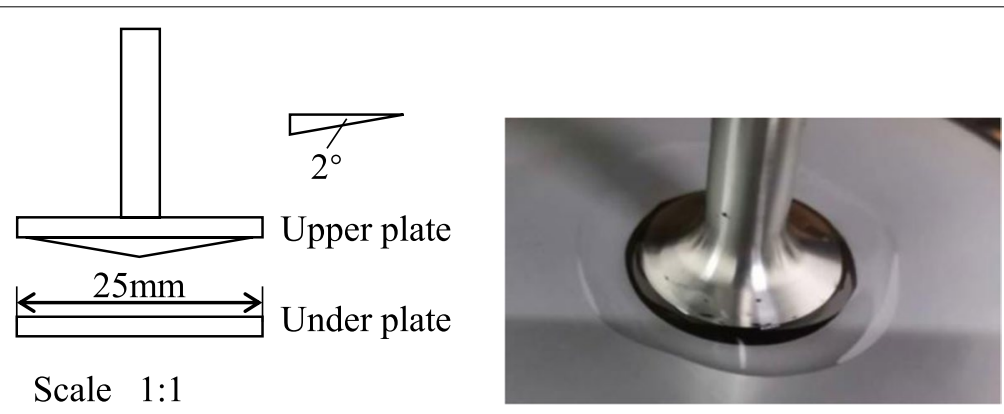

Fig. 1 The conical plate of rheology tests 
Table 3 Linear rheological region of the studied asphalt emulsions

\begin{tabular}{llll}
\hline Emulsions & $\mathbf{3 0} \mathrm{C}$ & $\mathbf{4 0}{ }^{\circ} \mathrm{C}$ & $\mathbf{5 0} \mathrm{C}$ \\
\hline $\mathrm{A}-40$ & $\leq 0.02 \mathrm{~Pa}$ & $\leq 0.01 \mathrm{~Pa}$ & $\leq 0.004 \mathrm{~Pa}$ \\
$\mathrm{~A}-50$ & $\leq 0.08 \mathrm{~Pa}$ & $\leq 0.05 \mathrm{~Pa}$ & $\leq 0.03 \mathrm{~Pa}$ \\
$\mathrm{~A}-60$ & $\leq 0.1 \mathrm{~Pa}$ & $\leq 0.08 \mathrm{~Pa}$ & $\leq 0.05 \mathrm{~Pa}$ \\
$\mathrm{~B}$ & $\leq 0.03 \mathrm{~Pa}$ & - & - \\
\hline
\end{tabular}

Interfacial tension of asphalt emulsions was measured using an A1200 interface tension meter. First, a platinum ring with a circumference of $60 \mathrm{~mm}$ was immersed $2 \sim 3 \mathrm{~mm}$ below the surface of asphalt emulsions. Then slowly lift up the platinum ring, and a membrane will be formed between the ring and the surface of asphalt emulsions. The maximum tension between the platinum ring and asphalt emulsions is the interfacial tension. The temperature of the experiment was $30^{\circ} \mathrm{C}$.

\section{Results and discussion}

Test method of rheological properties of asphalt emulsions Necessity analysis of preshear

Time sweep tests were conducted on ten parallel samples of asphalt emulsion A-40. The dynamic modulus and phase angle of different samples were shown in Fig. 3. It can be seen in Fig. 3a and b that the dynamic rheological test results of ten parallel samples are totally different. Studies showed that the rheology behavior of emulsions was affected by its mechanical history [15, 17]. The results in Fig. 3 proved that the effect of mechanical history on asphalt emulsions cannot be ignored. Preshear is an effective method to eliminate this effect. After preshear, the microstructure of emulsions was break down and the mechanical history can be considered as fully erased. Then, the effect of mechanical history was eliminated and reproducible results can be obtained. Thus, it is necessary to preshear asphalt emulsions before testing dynamic rheological properties.

\section{Preshear protocol}

A preshear protocol indicates that the emulsion was presheared for a certain preshear time at certain preshear rate. The method of determining the preshear rate and preshear time was discussed in this part. Asphalt emulsions A-40 and B, whose solid content is the same, were employed to investigate the suitable range of preshear rate and preshear time. Firstly, the viscosity versus shear rate of asphalt emulsions A-40 and B were tested, as shown in Fig. $4 \mathrm{a}$ and $\mathrm{b}$, respectively. It can be seen in Fig. 4a that the viscosity of asphalt emulsion A-40 decreases with the increase in shear rate until $2000 \mathrm{~s}^{-1}$. After $2000 \mathrm{~s}^{-1}$ the viscosity increases slightly and then keeps decreasing. Meanwhile, the asphalt emulsion A-40 demulsify at $2000 \mathrm{~s}^{-1}$, as shown in Fig. 5a and b. Thus, the preshear rate of asphalt emulsions A-40 should be smaller than $2000 \mathrm{~s}^{-1}$. It can be seen in Fig. $4 \mathrm{~b}$ that the viscosity of asphalt emulsion $B$ changes from decrease to increase at $2500 \mathrm{~s}^{-1}$. It can be speculated that the asphalt emulsion B begins to demulsify after $2500 \mathrm{~s}^{-1}$. However, the phenomenon of demulsification was not observed in the test sample of asphalt emulsion B, as shown in Fig. 5c and d. According to Table 2, the viscosity of asphalt emulsion B is high. Therefore, the asphalt droplets did not accumulate at the edge of plate. In summary, the demulsification of asphalt emulsions can be determined by viscosity curve or sample state. Based on above analysis, the suitable preshear rate for the studied asphalt emulsions is less than $2000 \mathrm{~s}^{-1}$. The preshear rate should be selected in the suitable range. If the shear rate is too low, it will take quite a long time to make

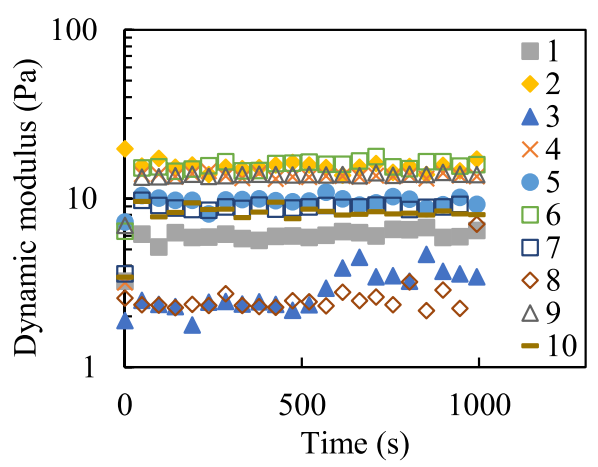

(a) Storage and Loss modulus

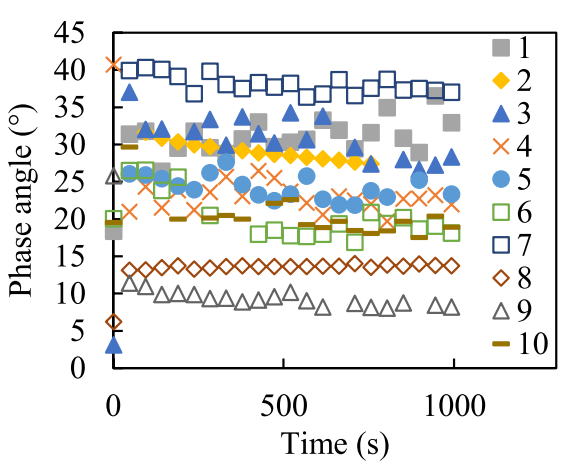

(b) Phase angle

Fig. 3 Rheology test results of asphalt emulsion A-40 


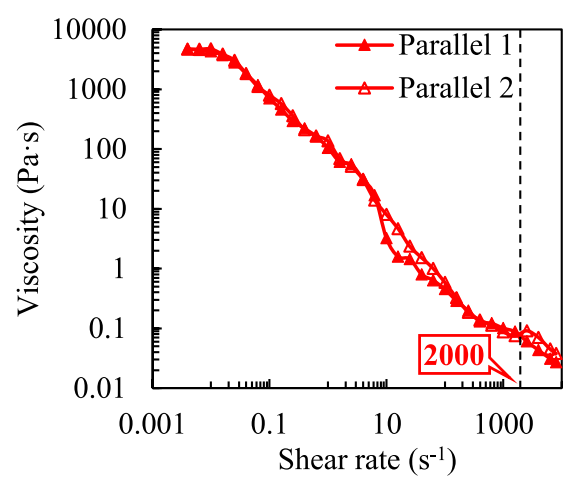

(a) Asphalt emulsion A-40

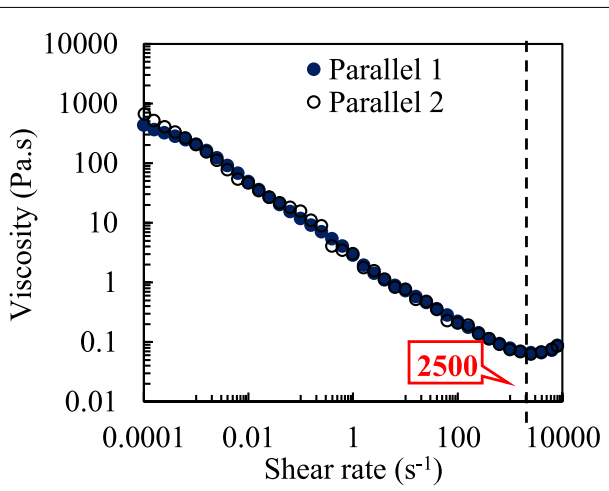

(b) Asphalt emulsion B

Fig. 4 Viscosity of the studied asphalt emulsions

the asphalt emulsions reach a uniform and stable state. Therefore, the suggested range of values is from $500 \mathrm{~s}^{-1}$ to $2000 \mathrm{~s}^{-1}$. Finally, $1000 \mathrm{~s}^{-1}$ was chosen as preshear rate for next study of preshear time.

Under the preshear rate of $1000 \mathrm{~s}^{-1}$, the viscosity versus shear time of the studied asphalt emulsions were shown in Fig. 6. It can be seen in Fig. 6a that the viscosity of asphalt emulsion A-40 remains stable after $35 \mathrm{~s}$, which indicates that the mechanical history of asphalt emulsions was eliminated and the droplets in asphalt emulsions reach a stable state. Thus, the suitable preshear time of asphalt emulsion A-40 should be longer than $35 \mathrm{~s}$. A similar phenomenon can also be seen in Fig. 6b. The viscosity of asphalt emulsion B decreases with the increase in shear time until $30 \mathrm{~s}$ and then keeps constant. It means that the suitable preshear time of asphalt emulsion B should be longer than $30 \mathrm{~s}$. Based on above analysis, the preshear time of the studied asphalt emulsions should be longer than $35 \mathrm{~s}$. Finally, the recommended value of preshear time was $35 \mathrm{~s}$ for the studied asphalt emulsions.

\section{Effect of recovery time on rheology tests}

Storage and loss modulus of asphalt emulsions A-40 and $\mathrm{B}$ after preshear were shown in Figs. 7a and 8a, respectively. It can be seen that the test results of parallel samples after preshear varies obviously. The effect of residual stress after preshear cannot be ignored and the recovery time is necessary. Among parallel samples, there is a reasonable variation range of storage/loss modulus. If

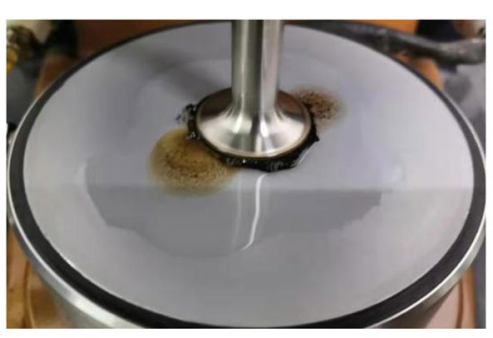

(a) A-40 at $2000 \mathrm{~s}^{-1}$

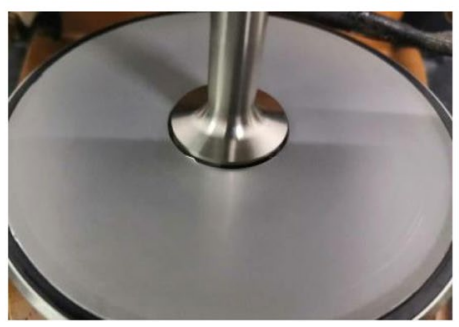

(c) B at $2500 \mathrm{~s}^{-1}$

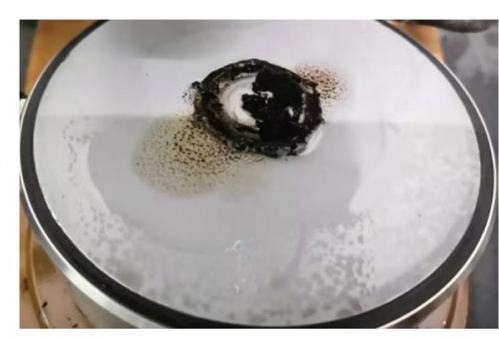

(b) A-40 at the end of the test

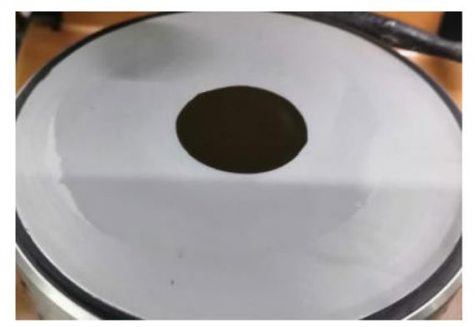

(d) B at the end of the test

Fig. 5 Samples at different shear rates 


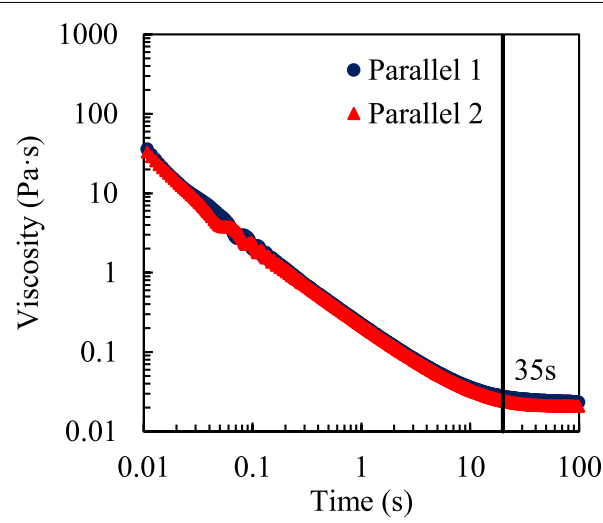

(a) Asphalt Emulsion A-40

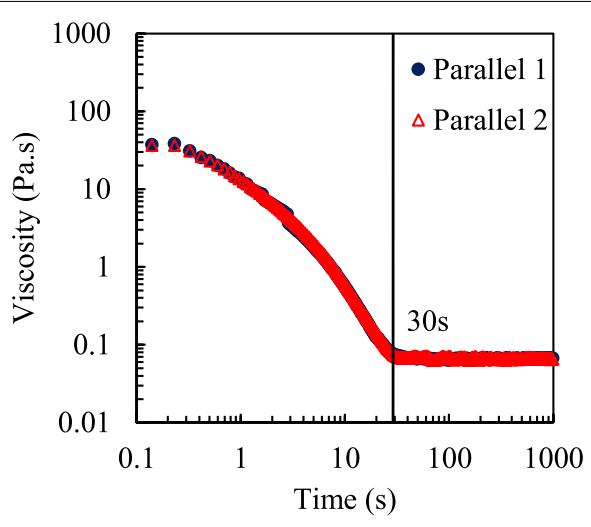

(b) Asphalt Emulsion B

Fig. 6 Viscosity Curves of Asphalt Emulsion A-40 and B at $1000 \mathrm{~s}^{-1}$

the storage/loss modulus are within this variation range, the test results are acceptable. The upper and lower limit value of the reasonable variation range were calculated by SPSS 21.0.

The two solid/dotted lines in Figs. 7 and 8 are the upper limit and lower limit of storage/loss modulus.
It can be seen in Fig. 7 that the suitable recovery time of asphalt emulsion A-40 ranges from 35 to $55 \mathrm{~s}$. The results in Fig. 8 showed that the suitable recovery time of asphalt emulsion B ranges from 20 to $60 \mathrm{~s}$. Within the range of suitable recovery time, a reproducible initial state of asphalt emulsions can be obtained. If

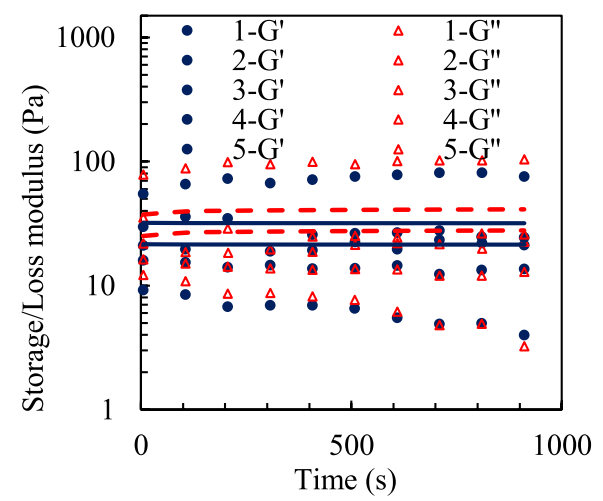

(a) $30 \mathrm{~s}$

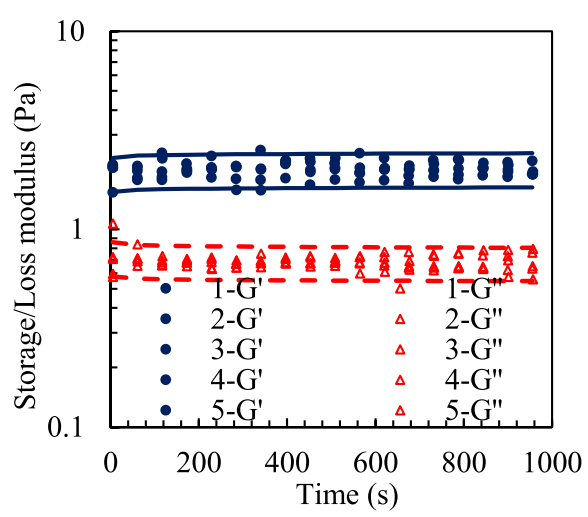

(c) $55 \mathrm{~s}$

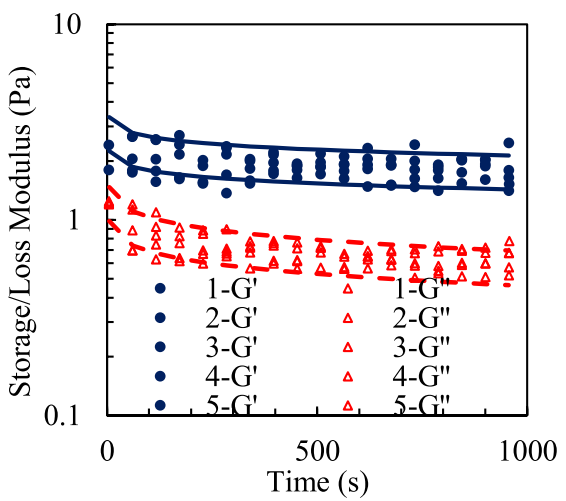

(b) $35 \mathrm{~s}$

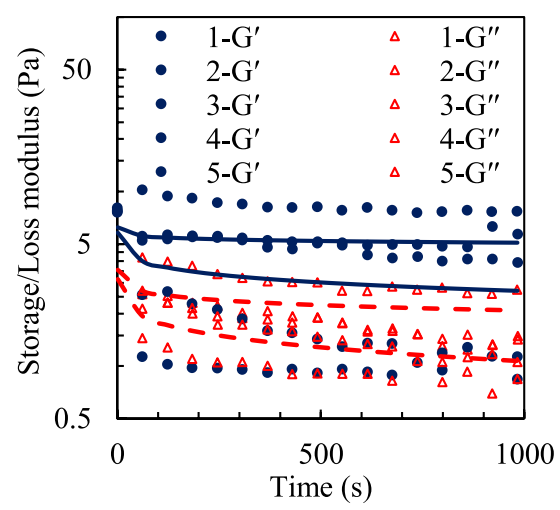

(d) $60 \mathrm{~s}$

Fig. 7 Parallel results of asphalt emulsion A-40 with different recovery times 


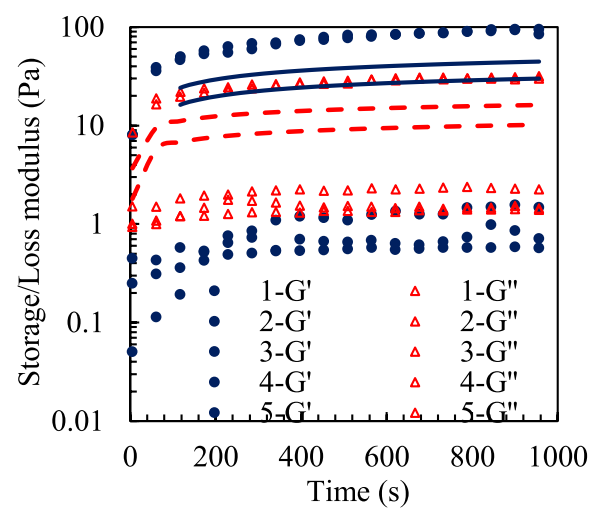

(a) $0 \mathrm{~s}$

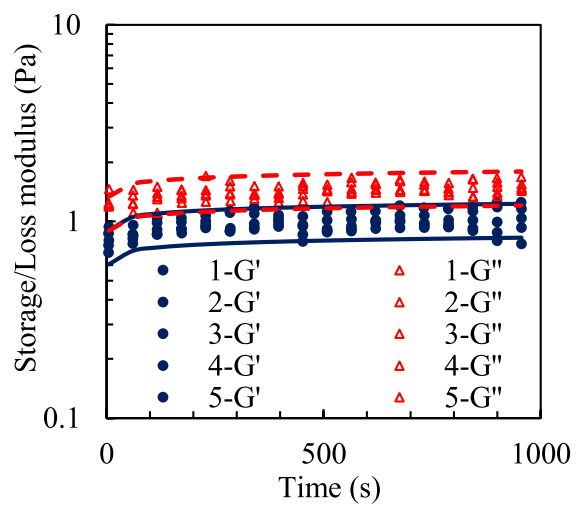

(c) $60 \mathrm{~s}$

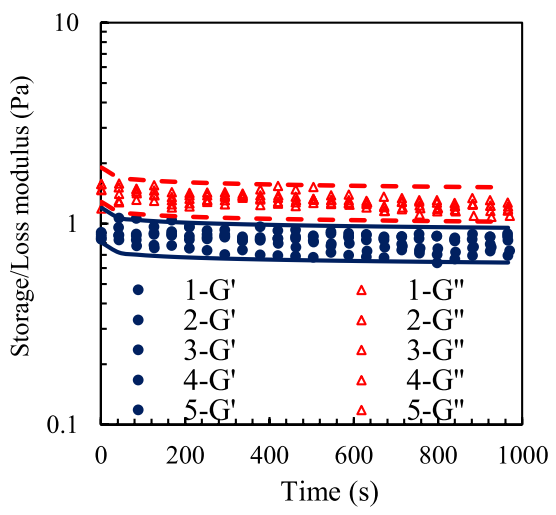

(b) $20 \mathrm{~s}$

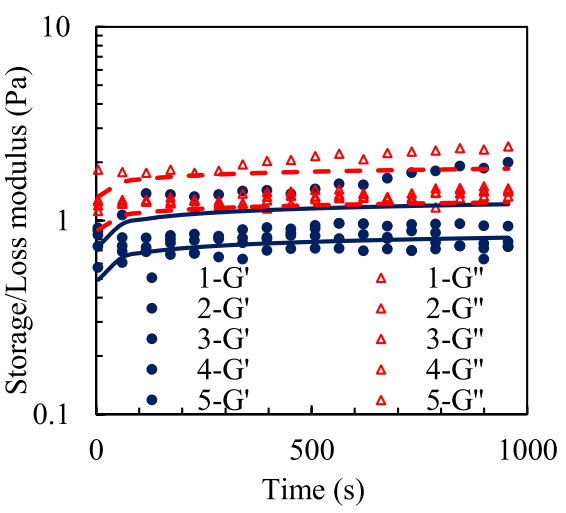

(d) $80 \mathrm{~s}$

Fig. 8 Parallel results of asphalt emulsion B with different recovery times

the recovery time is too short, the residual stress of preshear still exists, as shown in Figs. $7 \mathrm{a}$ and $8 \mathrm{a}$. If the recovery time is too long, the phenomenon of droplet aggregation and flocculation will appear again. The results will also be affected, as shown in Figs. $7 d$ and $8 \mathrm{~d}$. It cannot be ignored that the range of recovery time of different asphalt emulsions are different. The types of asphalt and emulsifier between different asphalt emulsions are different. Furthermore, the microscopic properties such as droplet size distribution, interfacial tensions are also different. Thus, the suitable range of recovery time of different asphalt emulsions are different. Finally, $40 \mathrm{~s}$ is chosen as the recommended recovery time for the studied asphalt emulsions in this study.

\section{Rheological properties of asphalt emulsions Static rheological properties of asphalt emulsions}

The relationship between shear stress versus shear rate were shown in Fig. 9. The Herschel-Bulkley model were employed to fit the curves in Fig. 9. The equations of modified Herschel-Bulkley model was shown in Eq. 1,

$$
\tau=\tau_{0}+k \dot{\gamma}^{\mathrm{n}}
$$

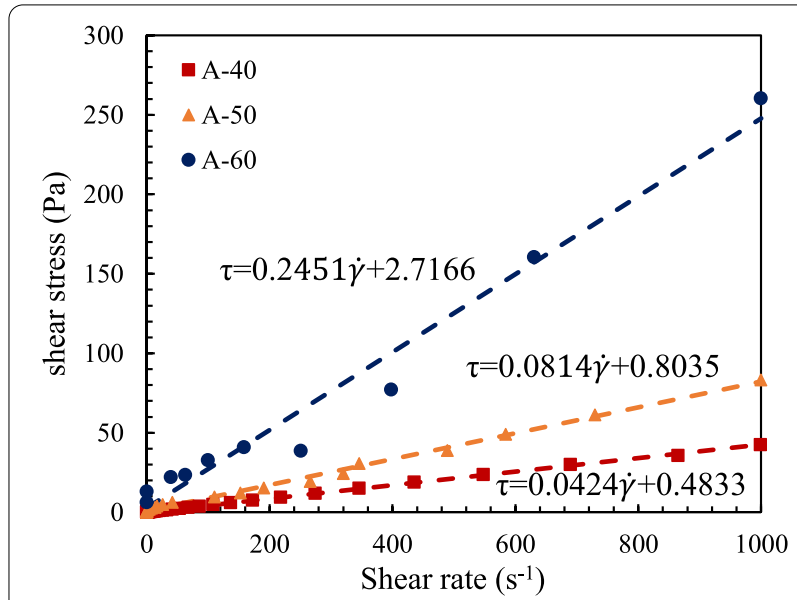

Fig. 9 Relationship between shear rate and shear stress of the studied emulsions 
where $\mathrm{\tau}$ is shear stress, $\mathrm{Pa} ; \mathrm{\tau}_{0}$ is yield shear stress, $\mathrm{Pa} ; \dot{\gamma}$ is shear rate, $\mathrm{s}^{-1} ; k$ is consistency index, $\mathrm{Pa} \cdot \mathrm{s}^{\mathrm{n}} ; n$ is flow behavior index, dimensionless.

It can be seen that the asphalt emulsions A-40, A-50 and A-60 behave as Bingham fluid. They show elastic characteristic when the shear stress is less than yield stress. The shear stress increases with the increase in shear rate when the shear stress is larger than yield stress. Morever, the yield stress increases with the increase in solid content. That means that the asphalt emulsion with high solid content is more hard to flow.

The viscosity of asphalt emulsions A-40, A-50 and A-60 were shown in Fig. 10. The viscosity of aphalt emulsions decreases with the increase in shear rate. Meanwhile, the viscosity of asphalt emulsions increases with the increase in solid content. Cross model was employed to fit the viscosity curves of asphalt emulsions. The equation of Cross model was shown in Eq. 2:

$$
\eta^{*}=\frac{\eta_{0}-\eta_{\infty}}{1+(k \omega)^{m}}+\eta_{\infty}
$$

where $\eta_{0}$ is zero shear viscosity, Pa; $\eta_{\infty}$ is viscosity when shear rate is $\infty, \mathrm{Pa} ; \eta^{*}$ is test viscosity, $\mathrm{Pa}$; $\omega$ is frequency, $\mathrm{Hz} ; k$ and $m$ are material constants.

The fitted curves of viscosity were also shown in Fig. 10. The test values and fitted values are almost coincidence which means that the Cross model can effectively characterize the viscosity of asphalt emulsions. The fitting parameters of Cross model were shown in Table 4. It can be concluded that zero shear viscosity of asphalt emulsions increases with the increase in solid content.

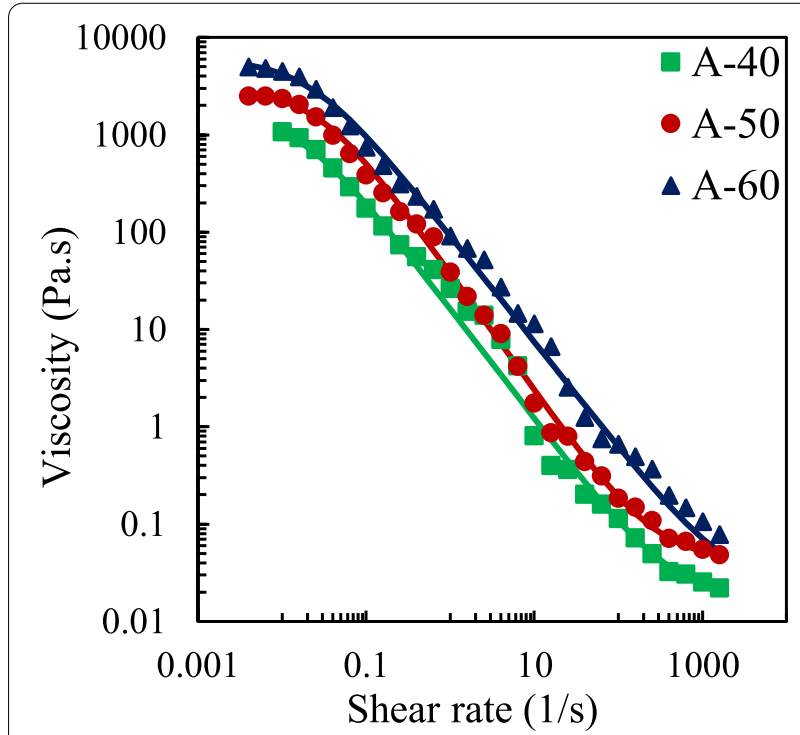

Fig. 10 Viscosity of studied asphalt emulsions with different solid content

\section{Dynamic rheological properties of asphalt emulsions}

Based on the rheology test method in Test method of rheological properties of asphalt emulsions section, dynamic rheological properties of the studied asphalt emulsions were tested after preshear. Figure 11 shows the storage and loss modulus of asphalt emulsions A-40, A-50 and A-60. The storage and loss modulus increase with the increase in frequency at different temperatures. Meanwhile, the storage and loss modulus increase with the increase in solid content, and their rate of increase is different.

The crossover point of storage and loss modulus curves was gel point, which represents the transform of asphalt emulsions from sol to gel. The gel point of the studied asphalt emulsions were shown in Fig. 12. The gel point increases with the increase in temperature and decreases with the increase in solid content. It means that asphalt emulsions are more prone to gel with the increase in solid content or with the decrease in temperature.

\section{Relationship between microscopic properties and rheological properties Microscopic properties of the studied asphalt emulsions}

Droplet size distribution of asphalt emulsions A-40, A-50 and A-60 were shown in Fig. 13. The droplet size distribution (DSD) curves reflect the mass distribution of different droplet size. The studied asphalt emulsions exhibit unimodal distribution. The shape of DSD curves of the studied asphalt emulsions is similar and the range of droplet size increases with the increase in solid content. The width of DSD curve of the studied asphalt emulsion increases with the increase in solid content. Based on the filling effect, the contact area of droplets in asphalt emulsion A-60 is larger than that in asphalt emulsions A-50 and A-40. The interacton force between droplets in asphalt emulsion A-60 also higher than that of others. Thus, the viscosity, storage modulus and loss modulus of asphalt emulsion A-60 are higher than that of asphalt emulsion A-50 and A-40. It should also be noted that the width of DSD curves of the studied asphalt emulsions is related to the rheological properties of asphalt emulsions. The wider the DSD curve, the higher the viscosity, storage modulus and loss modulus.

Table 4 Fitting parameters of Cross model

\begin{tabular}{llll}
\hline Parameters & A-40 & A-50 & A-60 \\
\hline$\eta_{0}(\mathrm{~Pa})$ & 1676 & 2900 & 5878 \\
$\eta_{\infty}\left(10^{-3} \mathrm{~Pa}\right)$ & 18 & 43 & 40 \\
$\mathrm{k}$ & 61 & 36 & 41 \\
$\mathrm{~m}$ & 1.1 & 1.2 & 1.1 \\
\hline
\end{tabular}




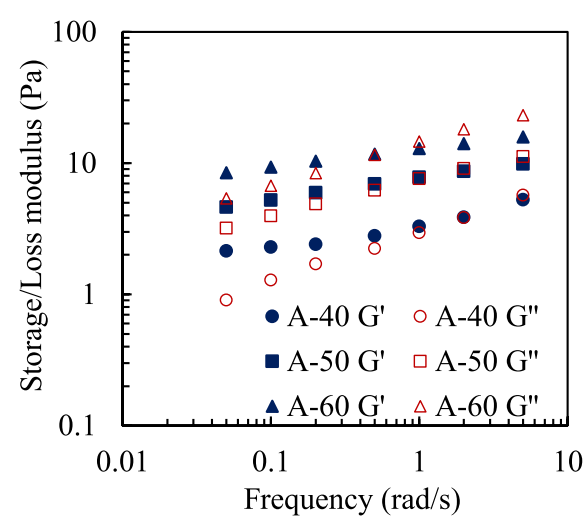

(a) $30^{\circ} \mathrm{C}$

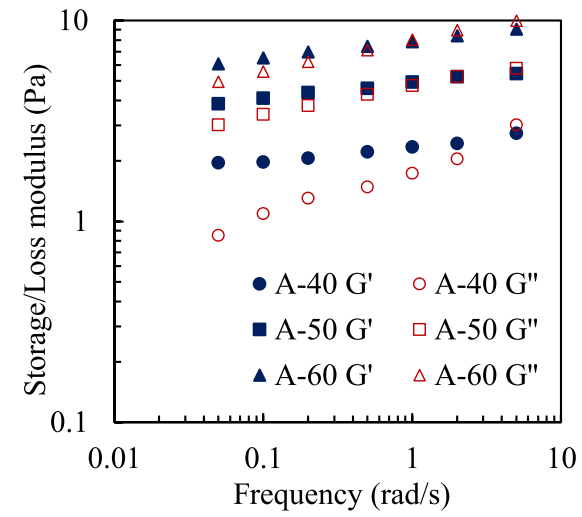

(b) $40^{\circ} \mathrm{C}$

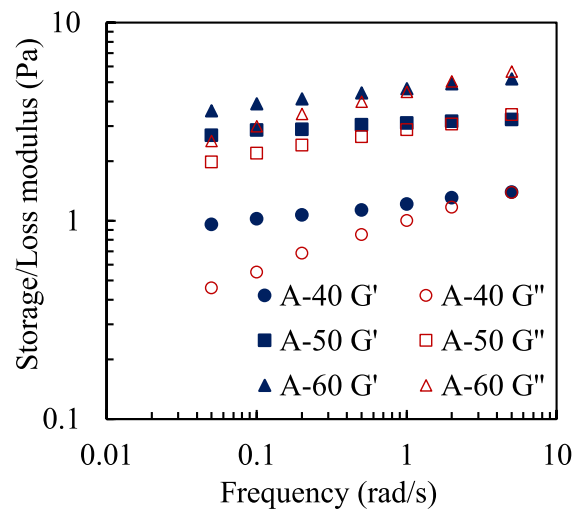

(c) $50{ }^{\circ} \mathrm{C}$

Fig. 11 Storage/loss modulus of asphalt emulsions

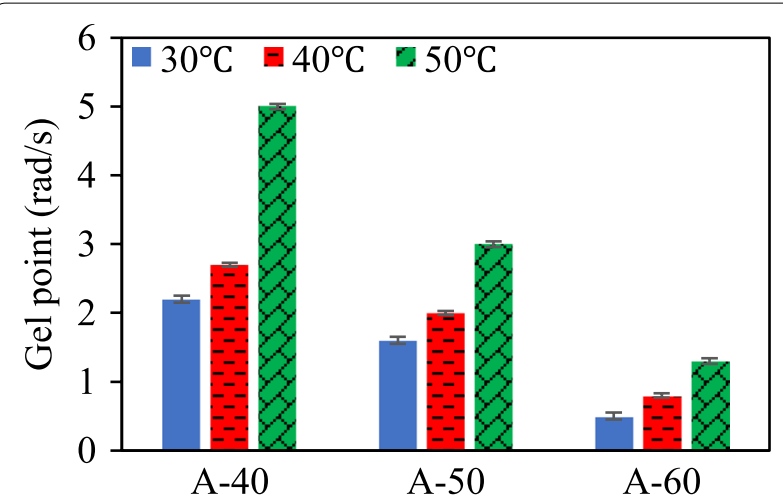

Fig. 12 Gel point of the studied asphalt emulsions

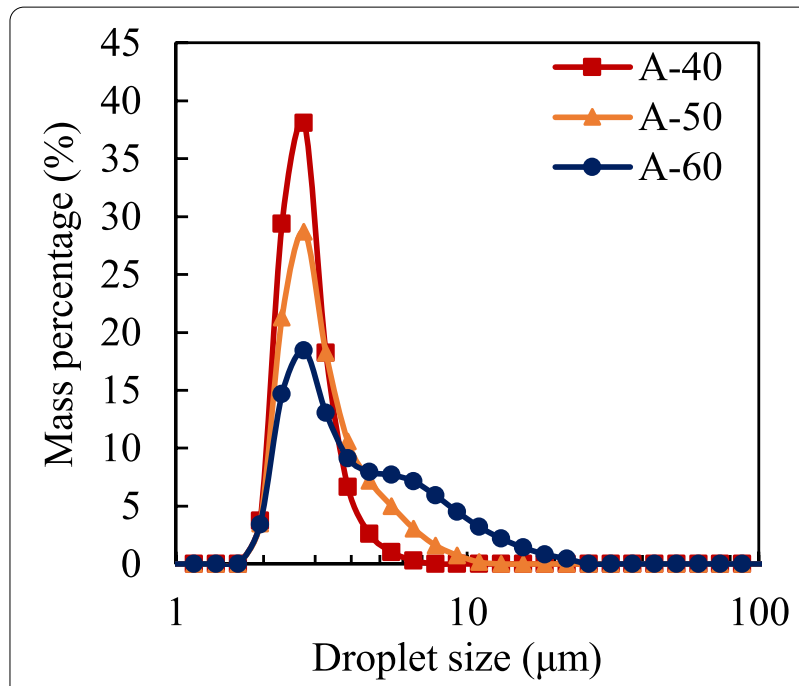

Fig. 13 Droplet size distribution of the studied asphalt emulsions 
In addition, the interfacial tensions also involved the fitting of storage and loss modulus in the following section. The interfacial tensions of A-40, A-50 and A-60 were shown in Table 5 . It can be concluded that the interfacial tensions of asphalt emulsions decrease with the increase in solid content. Interfacial tension is an important index to evaluate the stability of asphalt emulsions. The smaller the interfacial tension is, the more stable the interior of asphalt emulsions is. It can be concluded that the stability of asphalt emulsion A-60 is better than that of asphalt emulsions A-40 and A-50. Thus, for the studied asphalt emulsions, the stability increases with the increase in solid content.

\section{Relationship between microscopic properties and rheological properties}

The relationship between microscopic properties and rheological properties is characterized by palierne model in the field of emulsions. The palierne model was shown as follows:

$$
\begin{aligned}
& G^{*}(\omega)=G_{m}^{*}(\omega) \frac{1+3 \phi_{i} H_{i}(\omega)}{1-2 \phi_{i} H_{i}(\omega)} \\
& H_{i}(\omega)=\frac{4\left(\Gamma / d_{i}\right)\left[2 G_{m}^{*}(\omega)+5 G_{i}^{*}(\omega)\right]+\left[G_{i}^{*}(\omega)-G_{m}^{*}(\omega)\right] \times\left[16 G_{m}^{*}(\omega)+19 G_{i}^{*}(\omega)\right]}{40\left(\Gamma / d_{i}\right)\left[G_{m}^{*}(\omega)+G_{i}^{*}(\omega)\right]+\left[2 G_{i}^{*}(\omega)+3 G_{m}^{*}(\omega)\right] \times\left[16 G_{m}^{*}(\omega)+19 G_{i}^{*}(\omega)\right]}
\end{aligned}
$$

where $G^{*}(\omega)$ is dynamic modulus of asphalt emulsions, $\mathrm{Pa} ; G_{m}^{*}(\omega)$ is dynamic modulus of matrix phase, $\mathrm{Pa} ; G_{i}^{*}(\omega)$ is dynamic modulus of asphalt binders, $\mathrm{Pa} ; \Gamma$ is interfacial tension of asphalt binders, $\mathrm{mN} / \mathrm{m} ; d_{i}$ is average droplet size when the solid content is $\phi_{i}, \mu \mathrm{m}$; $\omega$ is frequency, $\mathrm{Hz}$.

Based on Palierne model, the dynamic modulus of asphalt emulsions was predicted by average droplet size and interfacial tension. The results were shown in Fig. 14. It can be seen that the difference between predicted results and test results is obvious. The palierne model cannot accurate characterize the relationship between dynamic modulus and average droplet size /interfacial tension.

It can be seen in Eq. 3 that only average droplet size $d_{i}$ was considered in palierne model and the distribution of emulsion droplets was ignored. Studies demonstrated that the difference between calculated value of palierne model and measured value is obvious [32]. To introduce the effect of droplet size distribution, the $\phi_{i}$ was replaced by equations of DSD curves in Table 4. Then $\phi_{i}=\int_{0}^{\infty} \phi(D) d D$.

Table 5 Interfacial tension of the studied asphalt emulsions

\begin{tabular}{lccc}
\hline Studied asphalt emulsions & A-40 & A-50 & A-60 \\
\hline Tested interfacial tension $\left(\mathrm{mN} \cdot \mathrm{m}^{-1}\right)$ & 2.43 & 2.01 & 1.89 \\
\hline
\end{tabular}

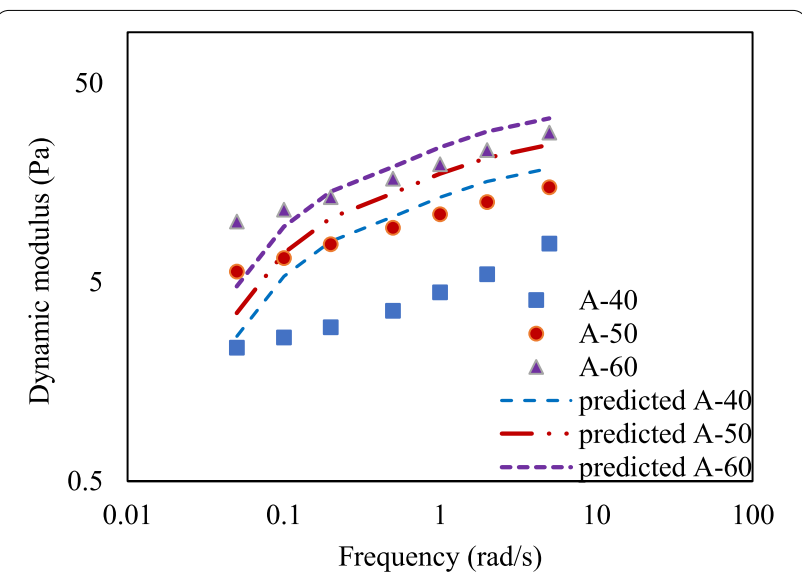

Fig. 14 Measured and predicted values of dynamic modulus by palierne model

Where $D$ is droplet size of asphalt emulsions, $\mu \mathrm{m}$; $\phi(D)$ is the fitting equations of droplet percentage versus droplet size.

Thus, the Eq. 3 can be written as:

$$
G^{*}(\omega)=G_{m}^{*}(\omega) \frac{1+3 \int_{0}^{\infty} H(\omega, D / \Gamma) \phi(D) d D}{1-2 \int_{0}^{\infty} H(\omega, D / \Gamma) \phi(D) d D}
$$

To simplify the calculation, this study uses $\hat{D}$ to replace $D / \Gamma$. Finally, the Eq. 5 can be written as follows:

$$
G^{*}(\omega)=G_{m}^{*}(\omega) \frac{1+3 \int_{-\infty}^{+\infty} H(\omega, \hat{D}) U(\hat{D}) \hat{D} d(\ln \hat{D})}{1-2 \int_{-\infty}^{+\infty} H(\omega, \hat{D}) U(\hat{D}) \hat{D} d(\ln \hat{D})}
$$

$$
H(\omega, \hat{D})=\frac{4\left[2 G_{m}^{*}(\omega)+5 G_{i}^{*}(\omega)\right] / \hat{D}+\left[G_{i}^{*}(\omega)-G_{m}^{*}(\omega)\right] \times\left[16 G_{m}^{*}(\omega)+19 G_{i}^{*}(\omega)\right]}{40\left[G_{m}^{*}(\omega)+G_{i}^{*}(\omega)\right] / \hat{D}+\left[2 G_{i}^{*}(\omega)+3 G_{m}^{*}(\omega)\right] \times\left[16 G_{m}^{*}(\omega)+19 G_{i}^{*}(\omega)\right]}
$$

In Eq. $6, U(\hat{D}) d \hat{D}=\Gamma \phi(\Gamma \hat{D}) d \hat{D}$

As $G^{*}(\omega)=G^{\prime}(\omega)+i G^{\prime \prime}(\omega)$, where $G^{\prime}(\omega)$ is storage modulus, $\mathrm{Pa} ; G^{\prime \prime}(\omega)$ is loss modulus, $\mathrm{Pa}$.

Thus,

$$
\begin{aligned}
& G^{\prime}(\omega)=\operatorname{Re}\left[G_{m}^{*}(\omega) \frac{1+3 \int_{-\infty}^{+\infty} H(\omega, \hat{D}) U(\hat{D}) \hat{D} d(\ln \hat{D})}{1-2 \int_{-\infty}^{+\infty} H(\omega, \hat{D}) U(\hat{D}) \hat{D} d(\ln \hat{D})}\right] \\
& G^{\prime \prime}(\omega)=\operatorname{lm}\left[G_{m}^{*}(\omega) \frac{1+3 \int_{-\infty}^{+\infty} H(\omega, \hat{D}) U(\hat{D}) \hat{D} d(\ln \hat{D})}{1-2 \int_{-\infty}^{+\infty} H(\omega, \hat{D}) U(\hat{D}) \hat{D} d(\ln \hat{D})}\right]
\end{aligned}
$$

where $d \hat{D}=\hat{D} d(\ln \hat{D})$.

Equations 6-9 are the modified palierne model which considers the droplet size distribution of asphalt emulsions. The microscopic characteristics tested in 
Microscopic properties of the studied asphalt emulsions section were employed to predicted the storage and loss modulus of asphalt emulsions by the modified palierne model. The test results and predicted results were shown in Fig. 15. It can be seen that the modified palierne model can effectively characterize the relationship between rheological properties and droplet size distribution of asphalt emulsions.

The study of the modified palierne model proved that the rheological properties of asphalt emulsions were determined by microscopic characteristics. During the production of asphalt emulsions, droplet size distribution of asphalt emulsions were heavily affected by shear rate, emulsifier content, modifier type, etc. Thus, the modified palierne model is helpful to the composition design and production process control of asphalt emulsions.

\section{Conclusion}

This study aims to investigate rheological properties of asphalt emulsions. The preshear protocol was determined and rheological properties of asphalt emulsions were tested after preshear. The microscopic characteristics The droplet size distribution of asphalt emulsions were tested, and the storage/loss modulus were predicted by the modified palierne model. Based on our results, the follow conclusions can be drawn:

1. Preshear before rheology tests of asphalt emulsions is necessary. Firstly, preshear rate was determined by curves of viscosity versus shear rate. Asphalt emulsions should not demulsify under the chosen preshear rate. Then, under the chosen preshear rate, preshear time was analyzed by curves of viscosity versus shear time. The suitable range of preshear

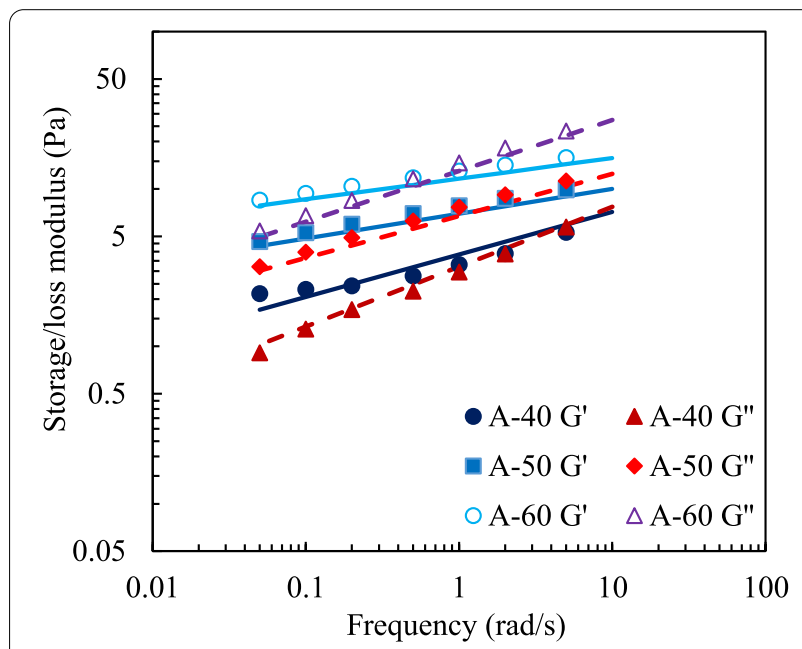

Fig. 15 Measured and predicted storage/loss modulus by modified palierne model time is the time when viscosity reaches the plateau. Finally, recovery time was determined by time sweep tests.

2. Asphalt emulsions behave as Bingham fluid at $30{ }^{\circ} \mathrm{C}$. Asphalt emulsions become hard to flow with the increase in solid content. The viscosity of asphalt emulsions increases with the increase in solid content.

3. The storage and loss modulus of asphalt emulsions increase with the increase in asphalt content. As the solid content increases or the temperature decreases, the asphalt emulsions are more prone to gel.

4. The rheological properties of asphalt emulsions were affected by droplet size distribution and interfacial tension. The prediction accuracy of the modified palierne model is higher than that of palierne model. Based on the modified Palierne model, the storage and loss modulus of asphalt emulsions can be accurately predicted by droplet size distribution and interfacial tension.

\section{Abbreviations}

A-40: Asphalt emulsion A with 40\% solid content; A-50: Asphalt emulsion A with $50 \%$ solid content; A-60: Asphalt emulsion A with $60 \%$ solid content; B40: Asphalt emulsion B with 40\% solid content; DSD: Droplet size distribution.

\section{Acknowledgements}

The corresponding author is grateful to the National Natural Science Foundation of China: [51778195, 51978218]. The authors want to express our gratitude to Weixiang Sun for his help in determination of preshear protocol.

\section{Authors' contributions}

All authors read and approved the final manuscript.

\section{Funding}

This work was supported by National Natural Science Foundation of China: [51778195, 51978218].

Availability of data and materials

All data generated or analyzed during this study are included in this published article.

\section{Declarations}

\section{Competing interests}

The authors declare that they have no competing interests.

Received: 30 May 2021 Accepted: 8 July 2021

Published online: 07 September 2021

References

1. Shaowen Du (2017) Effect of curing conditions on properties of cement asphalt emulsion mixture. Constr Build Mater 164:84-93

2. Ouyang J, Tan Y, Li Y et al (2015) Demulsification process of asphalt emulsion in fresh cement-asphalt emulsion paste. Mater Struct 48(12):3875-3883

3. Carrera V, Cuadri AA, Garcia-Morales M et al (2015) The development of polyurethane modified bitumen emulsions for cold mix applications. Mater Struct 48(10):3407-3414 
4. Ronald M, Luis FP (2016) Asphalt emulsions formulation: state-of-the-art and dependency of formulation on emulsions properties. Construct Build Mater 123:162-173

5. You L et al (2020) Stability and rheology of asphalt-emulsion under varying acidic and alkaline levels. J Clean Prod 256:120417

6. Yuliestyan A, García-Morales M, Moreno E et al (2017) Assessment of modified lignin cationic emulsifier for bitumen emulsions used in road paving. Mater Des 131(5):242-251

7. Mercado RA, Sadtler V, Marchal P et al (2012) Heteroflocculation of a cationic oil-in-water emulsion resulting from Fontainebleau's sandstone powder addition as a model for asphalt emulsion breakup. Ind Eng Chem Res 51:11688-11694

8. Liu M, Han S, Wang Z et al (2019) Performance evaluation of new waterborne epoxy resin modified emulsified asphalt micro-surfacing. Constr Build Mater 214:93-100

9. Ruien Yu, Zhu X, Zhou X et al (2018) Rheological properties and storage stability of asphalt modified with nanoscale polyurethane emulsion. Pet Sci Technol 36(1):85-90

10. Sarkar MTA, Rahman MN, Elsifei MA et al (2020) Rheological and molecular characterizations of tire rubber modified asphalt emulsion. Transp Res Rec 2674(3):12-26

11. Shirkavand Hadavand B et al (2020) Modified bitumen emulsion by anionic polyurethane dispersion nanocomposites. Road Mater Pavement Des 21(6):1763-1774

12. Ouyang J et al (2018) Effect of cement on the demulsifying behavior of over-stabilized asphalt emulsion during mixing. Constr Build Mater 177:252-260

13. Ouyang J, Tan Y (2015) Rheology of fresh cement asphalt emulsion pastes. Constr Build Mater 80:236-243

14. Ouyang J et al (2016) The role and interaction of superplasticizer and emulsifier in fresh cement asphalt emulsion paste through rheology study. Constr Build Mater 125:643-653

15. Xiomara G, Felix S, Manuel C et al (2002) Bitumen-in-water emulsions: an overview on formation, stability, and rheological properties. J Dispersion Sci Technol 23(1-3):405-418

16. Pignon F, Magnin A, Piau JM (1996) Thixotropic colloidal suspensions and flow curves with minimum: Identification of flow regimes and rheometric consequences. J Rheol 40(4):573-587

17. Weixiang S, Yanrui Y, Tao W et al (2012) Effect of adsorbed poly (ethylene glycol) on the gelation evolution of Laponite suspensions: aging time-polymer concentration superposition. J Colloid Interface Sci 376(1):76-82

18. Rogers SA, Vlassopoulos D, Callaghan PT (2008) Aging, yielding, and shear banding in soft colloidal glasses. Phys Rev Lett 100(12):128304

19. Rogers SA, Callaghan PT (2010) Time-dependent rheology of colloida star glasses. J Rheol 54(1):133-158

20. Gibaud T, Frelat D, Manneville S (2010) Heterogeneous yielding dynamics in a colloidal gel. Soft Matter 6:3482-3488

21. Conte T, Chaouche M (2016) Rheological behavior of cement pastes under large amplitude oscillatory shear. Cem Concr Res 89:332-344

22. Grenard V, Divoux T, Taberlet N et al (2014) Timescales in creep and yielding of attractive gels. Soft Matter 10:1555-1571

23. Romero N, Cárdenas A, Rivas H (2000) Creep compliance-time behavior and stability of bitumen in water emulsions. J Rheol 44(6):1247-1262

24. Nagata S (1975) Mixing: principles and applications. Halsted Press. https:// scholar.google.com/scholar_lookup?title=Mixing\%3A\%20Principles\% 20and\%20Applications\&publication_year=1975\&author=S.\%20Nagata.

25. Sherman P (2010) Emulsion science. London, New York. http://openl ibrary.org/b/OL5540461M/Emulsion science.

26. Gondreta P, Petit L (1997) Dynamic viscosity of macroscopic suspensions of bimodal sized solid spheres. J Rheol 41(6):1261-1274

27. Gingras J-P, Tanguy PA, Mariotti S et al (2005) Effect of process parameters on bitumen emulsions. Chem Eng Process 44(9):979-986

28. Mercado R, Fuentes $L$ (2017) Measure of asphalt amulsions stability by oscillatory rheology. Constr Build Mater 155:838-845

29. Palierne JE (1990) Linear rheology of viscoelastic emulsions with interfacial tension. Rheol Acta 29(3):204-214

30. Themeli A, Chailleux E, Farcas F et al (2016) Modeling the linear viscoelastic behavior of asphaltite-modified bitumens. Rheol Acta 55(11-12):1-13

31. Tan Y, Meng G (2014) Interfacial thickness and interaction between asphalt and mineral fillers. Mater Struct 47(4):605-614

32. Friedrich C, Gleinser W, Korat E et al (1995) Comparison of spheresize distributions obtained from rheology and transmission electron microscopy in PMMA/PS blends. J Rheol 39(6):1411-1425

\section{Publisher's Note}

Springer Nature remains neutral with regard to jurisdictional claims in published maps and institutional affiliations.

\section{Submit your manuscript to a SpringerOpen ${ }^{\circ}$ journal and benefit from:}

- Convenient online submission

- Rigorous peer review

- Open access: articles freely available online

- High visibility within the field

Retaining the copyright to your article

Submit your next manuscript at springeropen.com 